\title{
THE APPLICATION OF DIGITAL IMAGE CORRELATION TO INVESTIGATE THE HETEROGENEITY OF ACHILLES TENDON DEFORMATION AND DETERMINE ITS MATERIAL PARAMETERS ${ }^{1}$
}

\author{
Piotr Kohut, Krzysztof Holak, Martyna Ekiert, Andrzej MŁyniec \\ AGH University of Science and Technology, Department of Robotics and Mechatronics, Krakow, Poland \\ e-mail:pko@agh.edu.pl; holak@agh.edu.pl; mekiert@agh.edu.pl; mlyniec@agh.edu.pl
}

KRZYSZTOF A. TOMASZEWSKI

Andrzej Frycz Modrzewski Krakow University, Faculty of Medicine and Health Sciences, Krakow, Poland, and Scanmed St. Raphael Hospital, Krakow, Poland

e-mail:krtomaszewski@gmail.com

TADEusz UhL

AGH University of Science and Technology, Department of Robotics and Mechatronics, Krakow, Poland e-mail: tuhl@agh.edu.pl

The challenge for researchers is to develop measurement techniques that can deal with biological specimens (e.g. the human Achilles tendon) characterized by high deformation during examination. The relevant quantity which has to be investigated in laboratory experiments is the deformation or strain field of the specimen subjected to a given load. In experimental mechanics, the most remarkable technique used for strain field computation is the Digital Image Correlation (DIC) method. In the paper, the DIC method is employed to study biomaterial parameters of human Achilles tendons (AT) subjected to tensile uniaxial loadings. The application of DIC allows the heterogeneity of tendon deformation to be taken into consideration. Young's modulus of AT based on the strain field obtained from a vision-based measurement is estimated and the results are discussed. A map of Young's modulus (YM) is demonstrated as well.

Keywords: digital image correlation, full-field displacement, strain measurement, vision systems, human Achilles tendon, Young's modulus

\section{Introduction}

Achilles tendons (AT) are the graft materials employed in reconstructive orthopedics to repair ligaments, for example, anterior and posterior cruciate ligaments in the knee. However, biophysical properties of the human AT are not fully known - they vary in time, especially when subjected to mechanical or environmental factors (e.g. temperature, chemicals) (Maganaris et al., 2008). The estimation techniques of AT material parameters have still not been fully developed. Measurements of the elastic properties of tendons yield values of Young's modulus between $500 \mathrm{MPa}$ and $1850 \mathrm{MPa}$ (Wren et al., 2001). The values of the elastic modulus of tendon constituents are reported to be between $200 \mathrm{MPa}$ and $39000 \mathrm{MPa}$ depending on the measurement technique, spatial scale and strain rate (Młyniec et al., 2015a,b; Kahn et al., 2013). Young's modulus calculated on the basis of experimental results assumes homogeneous deformation of the tendon, which can be one of the sources of discrepancies. The inhomogeneous deformation of

\footnotetext{
${ }^{1}$ The paper summarizes the work which was presented at the 23rd International Conference on Computer Methods in Mechanics, PCM-CMM at Cracow, Poland, 8-12 September 2019
} 
AT during deformation results from the twisted structure of the tendon, as reported in (Pękala et al., 2017).

The examination of human soft tissue mechanical behavior is a challenging task, because the properties of biological materials are characterized by anisotropy, non-linearity and inhomogeneity. Many measurement methods have been described in the literature, both contact and non-contact ones, carried out in ex vivo, in situ or in vivo conditions. In the case of AT, one of the most important quantities investigated in laboratory experiments is its stiffness or Young's modulus. Classically, this material property is examined in a quasi-static tensile test in which both the specimen elongation or strain and the applied force are recorded. If a cross-sectional area of the tested material sample is known, Young's modulus can be obtained as a slope of the stress-strain characteristics graph in its linear region. In practice, contact sensors, like strain gages, extensometers or LVDT (Rashid et al., 2014) are still used. These measurement methods are simple and not expensive, but they have many drawbacks. They are invasive in nature and influence mechanical properties of the examined materials. Moreover, they always work as single-point gauges, which record strain in a set of discrete points. It is impossible to obtain full-field displacement or strain fields which are needed to investigate the inhomogeneity of the material stiffness.

Therefore, non-contact measurement methods have gained in importance and popularity. Among them, vision-based methods are employed frequently in experimental mechanics of biological tissue. For instance, in the paper (Takaza et al., 2013), passive skeletal muscle feedback to an impact load was analyzed in an experiment for the purpose of obtaining data for numerical inverse modeling. Each sample was marked with 9 dots on a sample surface prior to testing. The displacement of dots was computed by a tracker implemented in the MATLAB programming environment. The strain field was estimated based on measured displacements. The authors of (Untaroiu et al., 2015) investigated the injury response of human liver parenchyma subjected to a tensile load. A set of markers was painted on the surface of the tissue. During the tensile test, the displacement between the closest optical markers adjacent to the tear was tracked in the video until failure using the TEMA motion analysis software. The mechanical properties of human AT were investigated in a tensile test (Wren et al., 2001). A set of bead markers $1 \mathrm{~mm}$ in diameter were attached to the tendon surface at $1.0-1.5 \mathrm{~cm}$ intervals. The displacement of markers during elongation of the specimen was recorded by a camera.

One of the most commonly used non-contact measurement techniques in evaluation of mechanical properties of materials is the Digital Image Correlation (DIC) (Zappa and Hasheminejad, 2017). The method was originally developed for testing of engineering structural materials and spread to other fields of science and technology, also for material testing of specimens under high strain conditions (Hwang and Wu, 2012), such as polymers (Hwang et al., 2015) and biological and biologically inspired materials. In this method, the specimen is prepared before each examination by spraying a random texture on its surface. Then, its deformation under the applied load is recorded by one (in the case of 2D DIC) or two digital cameras (3D DIC or S-DIC, stereo DIC). The displacements of measurement points are computed based on two images of the specimen, the reference one and the image showing the sample under deformation. The strain field is obtained based on the displacement field. The DIC method became popular because of its simplicity, high accuracy and the ability to compute full-field displacement and strain data with the density of measurement points restricted only by the resolution of the image sensors used in the camera system.

The method was used for examination of mechanical behavior of porcine liver (Ahn and Kim, 2010) and skin tissue (Rizzuto et al., 2016). The 3D-DIC method was applied in investigations of spatial displacement fields of the bovine sclera (Myers et al., 2010) deformed under physiological and pathological pressure loads in a series of inflation tests. The mechanical properties of ligaments, such as elastic modulus and ultimate tensile strength, were examined in DIC-based 
uniaxial and bi-axial tests (Tan et al., 2015). The strain field of the entire human AT obtained using the DIC method was reported in (Luyckx et al., 2014). The method was also applied with dynamic ultrasound images to measure strain distribution in mice tendons in vivo (Okotie et al., 2012). Additionally, the DIC with ultrasound was applied in the measurement of lower limb muscle deformation (Affagard et al., 2014). The technique was applicable due to speckle patterns generated in ultrasound imaging. A broad review of applications of the DIC method in the biomechanical area was presented in (Palanca et al., 2016).

Due to the properties of human Achilles tendons, which are characterized by anisotropy and inhomogeneity, the application of non-contact measurement techniques seems to be the best solution. The demonstrated studies are a continuation of the research related to AT modeling and biomechanical parameter investigation (Młyniec et al., 2015a,b; Pękala et al., 2017; Obuchowicz et al., 2019).

The goal of this study is to investigate the inhomogeneity of stiffness of Achilles tendons. The DIC method for full-field displacement and strain computations have been applied and complemented by calculation of the local stiffness and Young's modulus for a selected set of vision data. The results show that values of Young's modulus of Achilles tendons must be considered with particular attention due to the inhomogeneity of AT stiffness which has rarely been taken into consideration in previously published papers. The methodology, results and discussion are presented below.

\section{Theoretical basis of the digital image correlation (DIC) measurement technique}

Digital image correlation is a vision-based method for analysis of strain or displacement fields. It requires a single camera (2D DIC) or a two camera system (3D DIC) and an illumination system. If the specimen does not have a sufficient natural random speckle pattern on its surface, it has to be prepared before an examination by covering a random speckle pattern on it. During a tensile test of the specimen, the load is constantly increased and, at the same time, a series of images of the specimen deformation is recorded by the camera. After the test, a computation of displacement and strain field is performed. The basic parameters of the method are specified: a region of interest (ROI), a grid of measurement points inside the ROI and the chosen size of the correlation patch. In each moment of time, a displacement field is computed based on pairs of images, the reference one and the one acquired after the application of a given load. In order to measure specimen displacement at one particular grid point, a square patch with a center pixel at the point of interest and a specified number of neighborhood pixels is constructed. The pixels in the patch are taken as a reference pattern that is tracked by the DIC algorithm over all the images of a sequence. For each successive image frame, the deformation model parameters are computed to optimize the applied similarity measure. The most general model is described as

$$
x_{i}^{\prime}=x_{i}+\xi\left(x_{i}, y_{i}\right) \quad y_{i}^{\prime}=y_{i}+\eta\left(x_{i}, y_{i}\right)
$$

where $\left(x_{i}, y_{i}\right)$ are the coordinates of the center of the image patch, and $\left(x_{i}^{\prime}, y_{i}^{\prime}\right)$ are the coordinates of the corresponding point in consecutive frames of a sequence, and the functions $\xi$ and $\eta$ describe the deformation model. The most frequently used in practice affine model is described as

$$
x_{i}^{\prime}=x_{i}+u_{x} \Delta x+u_{y} \Delta y \quad y_{i}^{\prime}=y_{i}+v_{x} \Delta x+v_{y} \Delta y
$$

The model has six parameters - a displacement vector $[u, v]$ for the center of the image patch and 4 parameters corresponding to a skew deformation of an image patch. The position of the image patch center on each frame of a sequence is computed based on the maximum value 
of the similarity criterion. The most important similarity criterion is the zero-normalized cross correlation coefficient $\left(C_{Z N C C}\right)$, delineated by (Chuda-Kowalska et al., 2015)

$$
C_{Z N C C}=\sum_{-M \leqslant i \leqslant M} \sum_{-M \leqslant j \leqslant M} \frac{\left(f\left(x_{i}, y_{j}\right)-f_{m}\right)\left(g\left(x_{i}, y_{j}\right)-g_{m}\right)}{\Delta f \Delta g}
$$

where

$$
\begin{aligned}
& f_{m}=\frac{1}{(2 M+1)^{2}} \sum_{-M \leqslant i \leqslant M} \sum_{-M \leqslant j \leqslant M} f\left(x_{i}, y_{j}\right) \\
& g_{m}=\frac{1}{(2 M+1)^{2}} \sum_{-M \leqslant i \leqslant M} \sum_{-M \leqslant j \leqslant M} g\left(x_{i}, y_{j}\right)
\end{aligned}
$$

and

$$
\begin{aligned}
\Delta f & =\sqrt{\sum_{-M \leqslant i \leqslant M} \sum_{-M \leqslant j \leqslant M}\left(f\left(x_{i}, y_{j}\right)-f_{m}\right)^{2}} \\
\Delta g & =\sqrt{\sum_{-M \leqslant i \leqslant M-M \leqslant j \leqslant M} \sum_{-M}\left(g\left(x_{i}, y_{j}\right)-g_{m}\right)^{2}}
\end{aligned}
$$

where $f, g$ denote image patch intensities for the reference and analyzed images, $M$ is the number of pixels in the patch in two perpendicular directions of the patch. The $C_{Z N C C}$ coefficient is optimized over 6 parameters with the application of Gauss-Newton's optimization method. $\varepsilon_{x x}, \varepsilon_{y y}$ and $\gamma_{x y}$ strain components are calculated by employing the second-order Green-Lagrange strain formula

$$
\begin{aligned}
\varepsilon_{x x} & =\frac{1}{2}\left(2 u_{x}+u_{x}^{2}+v_{x}^{2}\right) \quad \varepsilon_{y y}=\frac{1}{2}\left(2 v_{y}+u_{y}^{2}+v_{y}^{2}\right) \\
\gamma_{y y} & =\frac{1}{2}\left(u_{y}+v_{x}+u_{x} u_{y}+v_{x} v_{y}\right)
\end{aligned}
$$

The digital image correlation method yields full-field displacement and strain maps obtained in an optical passive measurement.

\section{Experimental investigation}

The experimental setup consisted of an Instron 8872 material testing machine and a developed vision system (Fig. 1). The architecture of the vision system was composed of a high frame rate Phantom v9.1 camera with a $50 \mathrm{~mm}$ focal length lens, a Dedocool lighting system and NCORR software. Image acquisition was performed with the frame rate of $100 \mathrm{fps}$ and image resolution of: $1632 \times 800$ at the exposure of $0.7 \mathrm{~ms}$. A Carl Zeiss Planar T* $50 \mathrm{~mm} \mathrm{~F} / 1.4$ lens with F/11 adjustment was mounted to the camera. The camera was oriented perpendicularly to the analyzed tendon surface. The Dedocool lighting system with two heads allowed up to $2 \mathrm{mln}$ lux per head. To express findings in metric units, a scale coefficient of the vision system was calculated based on the known distance between two points of standard length (a ruler).

\subsection{Sample collection and preparation}

The measurements were carried out on 4 human Achilles tendons (AT) including 2 male and 2 female samples (patients without any comorbidities). AT specimens, together with the calcaneal bone in the lower insertion area and muscle fragments in the upper insertion area, were dissected at the Department of Forensic Medicine (Jagiellonian University Medical College, 


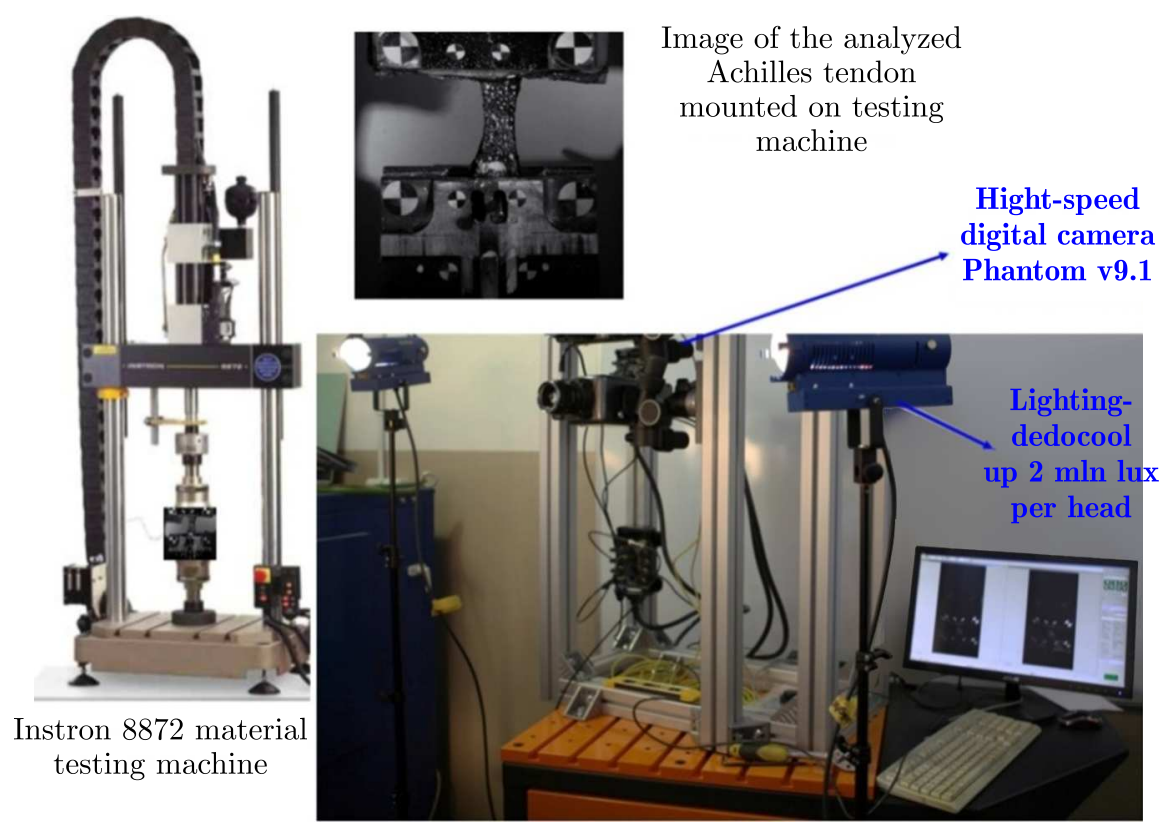

Fig. 1. Experimental setup

Krakow). All samples were obtained from cadavers up to 24 hours post-mortem, then frozen at $-80^{\circ} \mathrm{C}$ and transferred to the Department of Anatomy (Jagiellonian University Medical College, Krakow) for further studies. Four hours before the scheduled measurement time, the specimens were taken out of the freezer, wrapped into sterile gauzes moistened with saline solution and left at room temperature for 4 hours for thawing. The thawed tendons were cleaned of excess membranes as well as of gastrocnemius and soleus muscle fragments. The calcaneal bone in each specimen was drilled through in order to place a thin rod into it. Additionally, to obtain tracker points for the DIC approach, the front side of the specimens was stained black using Indian Ink and then dotted with a white paint. Regarding the field of view and image resolution, the proper size of a random speckle pattern should range from $0.7 \mathrm{~mm}$ up to $2.8 \mathrm{~mm}$. These requirements were fulfilled in the conducted tests.

\subsection{Biomechanical testing}

Biomechanical tests were carried out with a material testing machine (Instron 8872 model, INSTRON, Norwood, Massachusetts, USA) using a load cell with a maximum capacity of $10 \mathrm{kN}$ and a standard atmosphere of 23/50 conditions. The upper insertion area of the tested AT was clamped in grasping clamps with a sandpaper on the inside (to prevent slipping). The calcaneus bone of the tested AT was fixed in the lower clamps using the rod, previously placed into the heel. Then, the sample width and thickness were determined based on a set of images acquired by the camera. Samples were subjected to preloading with $40 \mathrm{~N}$ maintained for $1 \mathrm{~min}$. After that, tensile tests were performed using increasing uniaxial loading with a $50 \mathrm{~mm} / \mathrm{min}$ head displacement rate and displacement limited to $50 \mathrm{~mm}$. The slected low head displacement rate corresponds to the static loading of the tendon. The initiation of the testing run also triggered video system registration. After each run, the time evolution of the tensile load applied by the testing machine was saved for further calculations.

\subsection{Calculation of Achilles tendon stiffness}

The stiffness of the tested samples was evaluated in terms of the modulus of elasticity (Young's modulus), calculated from the slope of stress-strain curves. The stress component 
was determined by dividing the value of the load, obtained from the testing machine, by the tendon cross-section area. In order to estimate the cross-section area, it was assumed that it had an elliptical shape in which semi-major and semi-minor axes were equal to half of the sample width and thickness, respectively. The width and thickness were measured after the specimen was placed in the testing machine and subjected to preloading. In order to perform qualitative analysis of the stress field, the engineering stresses were selected and calculated as a force divided by the initial cross-sectional area of the sample. The strain $\varepsilon_{y y}$ component, calculated as a second-order Green-Lagrange strain, was determined based on the DIC approach. The computation of displacement and strain fields was carried out in the Open Source Ncorr v1.2 software. The program was developed in the MATLAB programming environment with computationally intensive algorithms optimized through the use of $\mathrm{C}++/ \mathrm{MEX}$. For each tested tendon, 10 stress-strain curves were obtained. For each one, the stress component remained the same, however, the strain component was calculated based on different sets of tracking points. For each plotted curve, a linear regression method was applied, and the line slope $a, y$-intercept $b$ and the coefficient of determination $R^{2}$ were taken as a result. Then, in order to ensure a high level of linear match, lines with $R^{2}<0.95$ were rejected for further analysis and the slopes of remaining fits were averaged. As a result, for each tested tendon, an averaged value of Young's modulus was obtained.

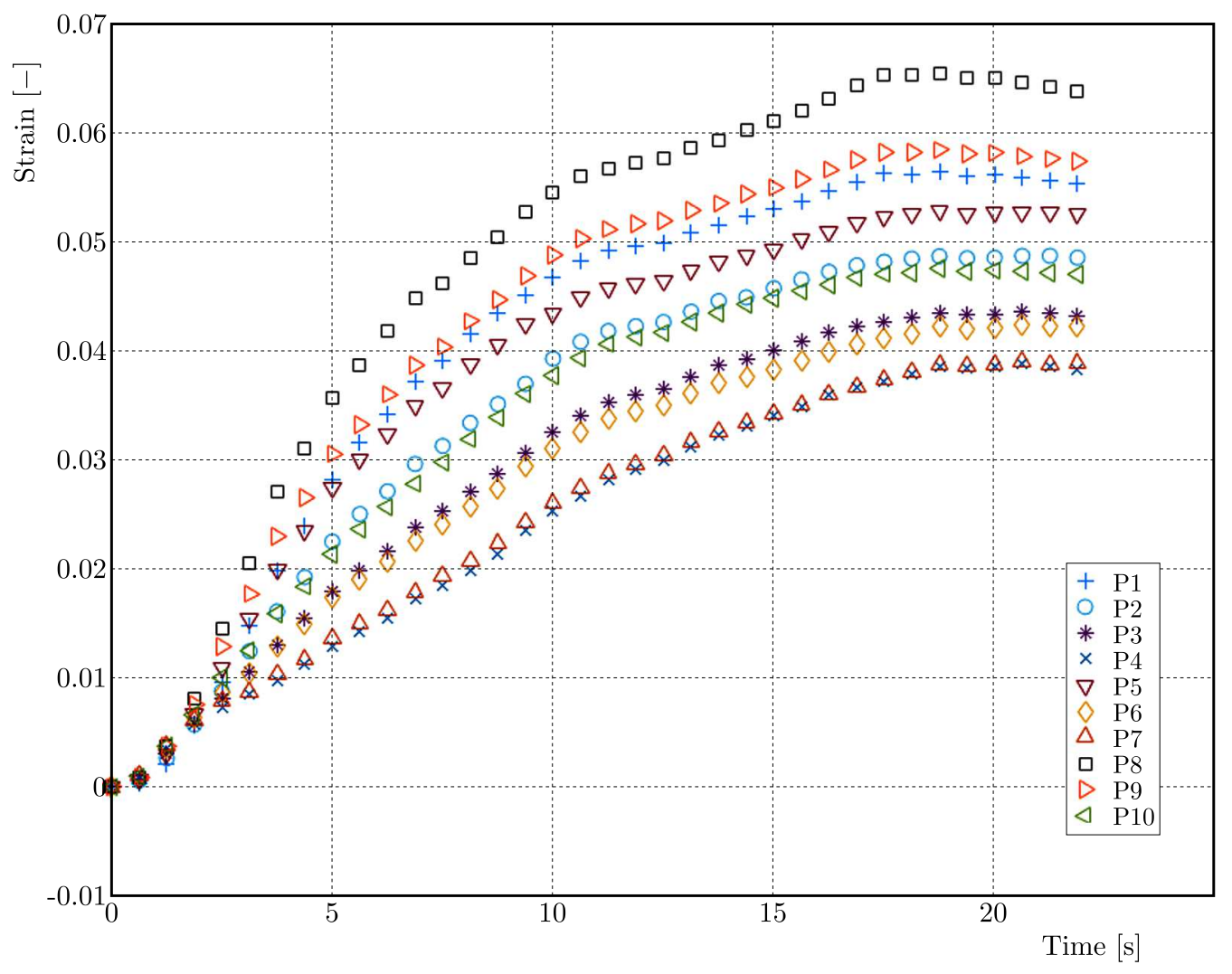

Fig. 2. Time evolution of the strain $\varepsilon_{y y}$ component during tensile tests for selected Achilles tendons

\section{Results and discussion}

An example of the stress-strain curves obtained for selected Achilles tendons (AT) is presented in Fig. 3. Following the scheme described in Section 3, linear regression coefficients were determined and Young's modulus of each tendon was calculated (Table 1, Fig. 4). The averaged value of Young's modulus for the tested samples was equal to $264.78 \pm 55.11 \mathrm{MPa}$ (Table 1) that is 
comparable with the values reported in (Chen et al., 2013). The values of the elastic modulus reported in literature are between $374.24 \mathrm{kPa}$ (Cao et al., 2018) and $2.0 \mathrm{GPa}$ (Kongsgaard et al., 2011) and depend on e.g. the cadaver age type of study (Kongsgaard et al., 2011) and strain rate (Wren et al., 2001). An increase of the specimen stiffness with the strain rate results from viscoelastic properties of the tissue that is caused by tissue structure.

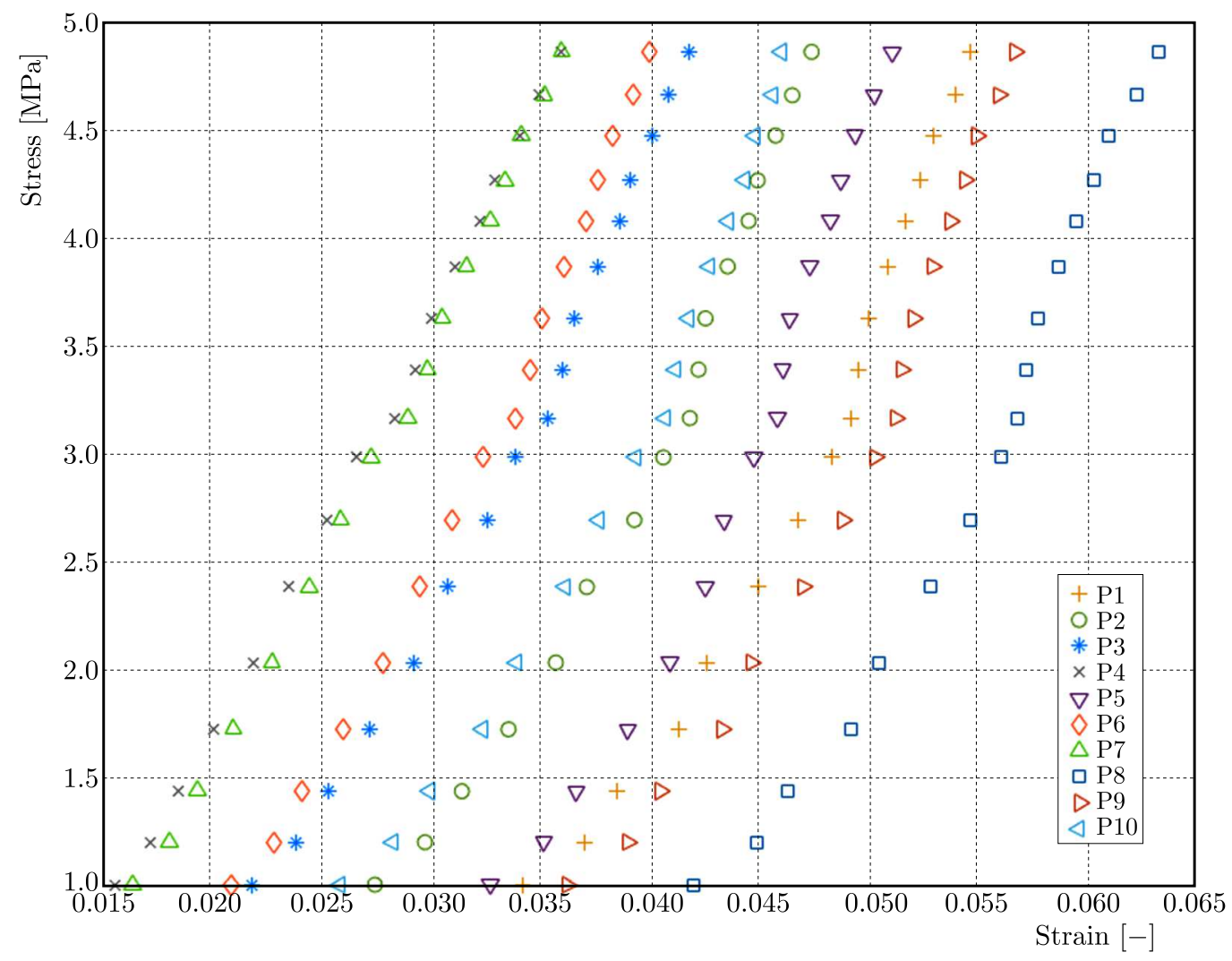

Fig. 3. Stress-strain curves for selected Achilles tendons. Each of the 10 curves relates to a different pair of points tracked by the vision system

Table 1. Young's modulus $E$ (identical with averaged line slope a together with deviation $u(E)$ calculated for 4 tested Achilles tendons (AT) and averaged for the entire tested group

\begin{tabular}{|c|c|c|c|c|c|c|c|}
\hline \multicolumn{2}{|c|}{ AT 1 } & \multicolumn{2}{c|}{ AT 2 } & \multicolumn{2}{c|}{ AT 3 } & \multicolumn{2}{c|}{ AT 4 } \\
\hline $\begin{array}{c}E \\
{[\mathrm{MPa}]}\end{array}$ & $\begin{array}{c}u(E) \\
{[\mathrm{MPa}]}\end{array}$ & $\begin{array}{c}E \\
{[\mathrm{MPa}]}\end{array}$ & $\begin{array}{c}u(E) \\
{[\mathrm{MPa}]}\end{array}$ & $\begin{array}{c}E \\
{[\mathrm{MPa}]}\end{array}$ & $\begin{array}{c}u(E) \\
{[\mathrm{MPa}]}\end{array}$ & $\begin{array}{c}E \\
{[\mathrm{MPa}]}\end{array}$ & $\begin{array}{c}u(E) \\
{[\mathrm{MPa}]}\end{array}$ \\
\hline \hline 175.08 & 37.60 & 203.66 & 11.78 & 391.02 & 85.32 & 289.36 & 85.73 \\
\hline \multicolumn{6}{|c|}{$\bar{E}_{\mathrm{AT} 1-\mathrm{AT} 4}=264.78 \pm 55.11 \mathrm{MPa}$} \\
\hline
\end{tabular}

A map representing Young's modulus inhomogeneity of Achilles tendons was calculated based on the full-field strain field and stress computed using the applied load with the assumption of a constant cross-section of each specimen. Examples of Young's modulus maps computed for one of the examined specimens are presented in Fig. 5.

\section{Conclusions}

Regarding various measurement techniques employed in the testing of biological materials, contactless measurement methods have belonged to the most promising group due to their many 


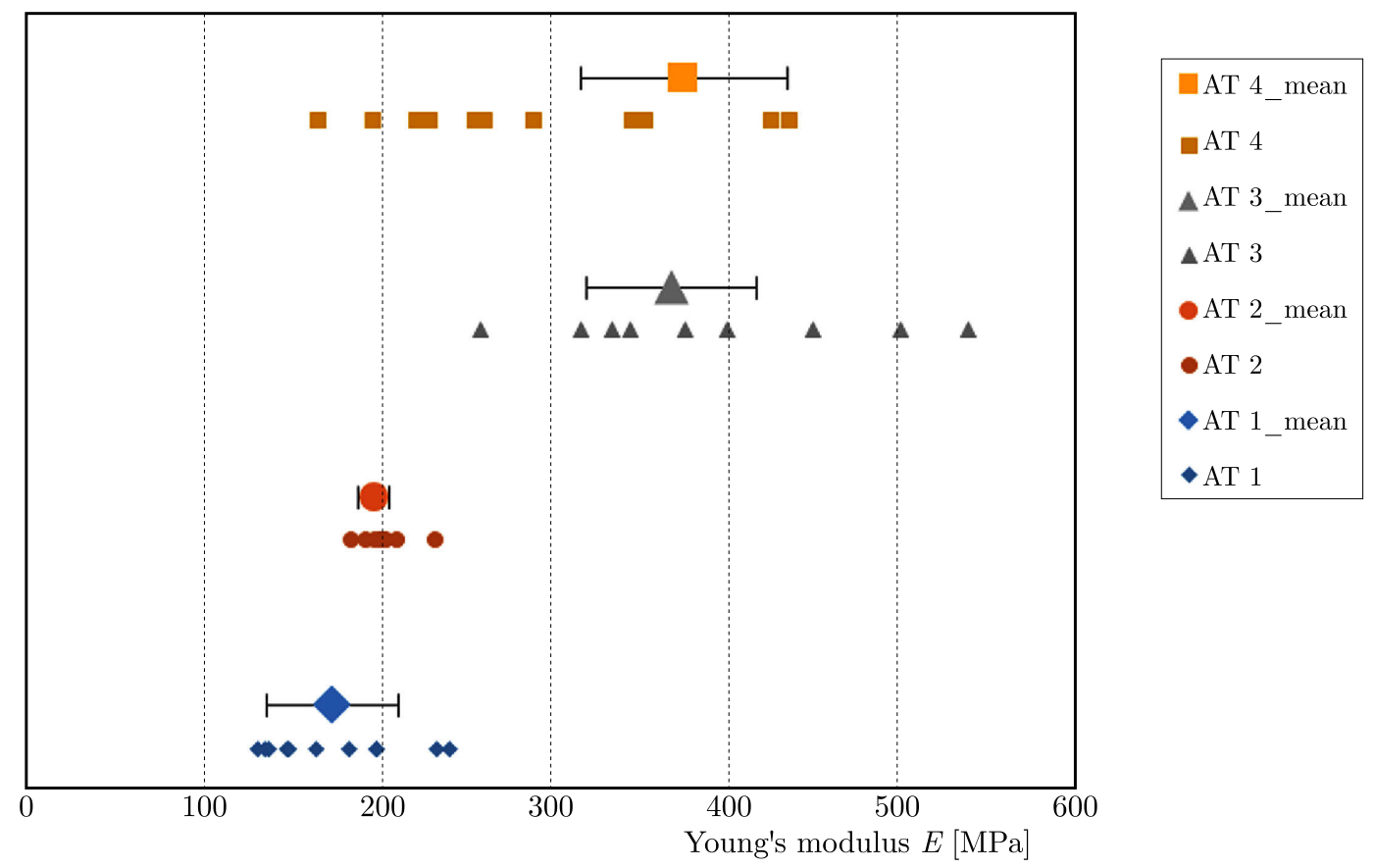

Fig. 4. Distribution of Young's modulus for all tested tendons

(a)

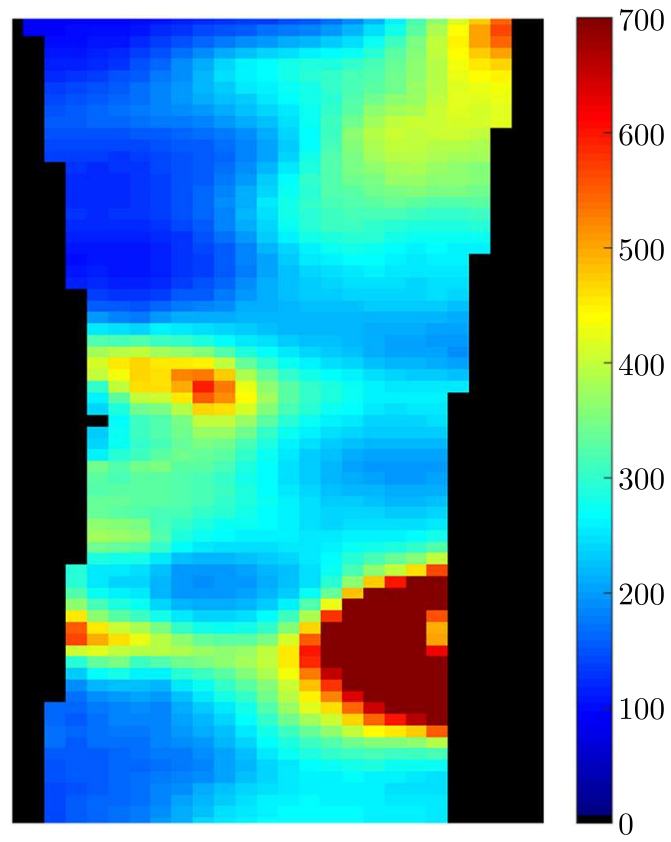

(b)

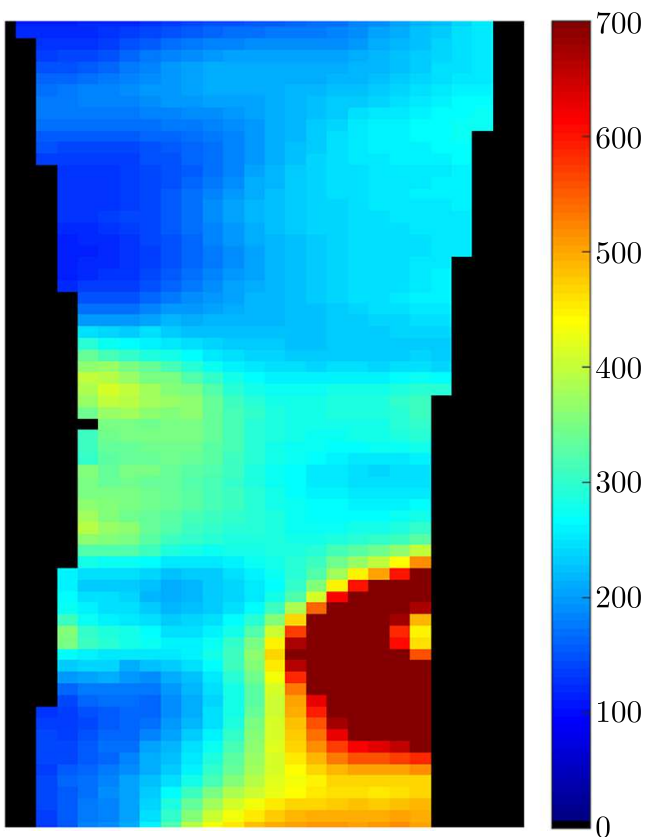

Fig. 5. Map of Young's modulus [MPa] of one of the examined specimen (AT 3), (a) Young's modulus map computed for 250 frame of the image sequence, (b) Young's modulus map computed for 350 frame of the image sequence

advantages. Undoubtedly, the lack of the influence on mechanical properties of the tested materials as well as final results in the form of displacement and strain fields have posed the greatest benefit of the DIC-based methods. In contrast to contact sensors which yield approximate mean values of data, the DIC technique allows one to determine the local strain and, thus, the results obtained by DIC methods are more reliable. It must be highlighted that the DIC method also allows the heterogeneity of the AT deformation to be taken into account. 
Furthermore, this method is straightforward to use and is characterized by uncomplicated preparation of a biological specimen surface applying random patterns by means of spray-painting.

The main advantage of the proposed solution relies on the way of estimating Young's modulus. Namely, by utilizing the set of selected and spread points of AT, sets of strain-stress curves were estimated and then the averaged Young's modulus was computed. In comparison with other approaches, the presented method resulted in lower errors while estimating the biomechanical parameters of biological structures. Furthermore, the inhomogeneity of AT was taken into account by employing DIC.

The computed map of Young's modulus is a function of space (position of measurement points on the tendon surface). It also changes with time, as the stretch of the tendon increases. The benefit of the YM map computed is the ability to identify specific areas in the specimen where the loss of stiffness can be observed as soon as it develops in mechanical examination.

Due to the observed heterogeneity of the twisted structure of a tendon, it is necessary to conduct research into a lower scale. Therefore, there is a need for further research in this area to investigate Young's modulus in each sub-tendon.

Acknowledgment

The research protocol was endorsed by the Jagiellonian University Medical College Ethics Committee (Protocol No. KBET/269/B/2011).

\section{References}

1. Affagard J.S., Bensamoun S.F., Feissel P., 2014, Development of an inverse approach for the characterization of in vivo mechanical properties of the lower limb muscles, Journal of Biomechanics, 136, 11

2. Aнn B., Kiм J., 2010, Measurement and characterization of soft tissue behavior with surface deformation and force response under large deformations, Medical Image Analysis, 14, 138-148

3. Cao W., Sun Y., Liu L., Wang Z., Wu J.Y., Qiu L., Wang Y.X., Yuan Y., Shen S.F., Chen Q., Chen T., Zhang W., Wu C.J., Liu F.X., Zhong S.G., Chen L., Tong M.H., Cui L.G., Guo R.J., 2018, A multicenter large-sample shear wave ultrasound elastographic study of the Achilles tendon in Chinese adults, Journal of Ultrasound in Medicine, 38, 5, 1191-1200

4. Chen X.M., Cui L.G., He P., Shen W.W., QIan Y.J., Wang J.R., 2013, Shear wave elastographic characterization of normal and torn Achilles tendons: A pilot study, Journal of Ultrasound in Medicine, 32, 3, 449-455

5. Chuda-Kowalska M., Gajewski T., Garbowski T., 2015, Mechanical characterization of orthotropic elastic parameters of a foam by the mixed experimental-numerical analysis, Journal of Theoretical and Applied Mechanics, 53, 2, 383-394

6. Hwang S.F., Shen M.C., Hsu B.B., 2015, Strain measurement of polymer materials by digital image correlation combined with finite-element analysis, Journal of Mechanical Science and Technology, 29, 10, 4189-4195

7. Hwang S.F., Wu W.J., 2012, Deformation measurement around a high strain-gradient region using a digital image correlation method, Journal of Mechanical Science and Technology, 26, 10, $3169-3175$

8. Kahn C.J., Dumas D., Arab-Tehrany E., Marie V., Tran N., Wang X., Cleymand F., 2013, Structural and mechanical multi-scale characterization of white New-Zealand rabbit Achilles tendon, Journal of the Mechanical Behavior of Biomedical Materials, 26, 81-90

9. Kongsgaard M., Nielsen C.H., Hegnsvad S., Aagaard P., Magnusson S.P., 2011, Mechanical properties of the human Achilles tendon, in vivo, Clinical Biomechanics, 26, 7, 772-777 
10. Luyckx T., Verstraete M., De Roo K., De Waele W., Bellemans J., Victor J., 2014, Digital image correlation as a tool for 3D strain analysis in human tendon tissue, Journal of Experimental Orthopaedics, 1, 7

11. Maganaris C.N., Narici M.V., Maffulli N., 2008, Biomechanics of the Achilles tendon, Disability and Rehabilitation, 30, 20-22, 1542-1547

12. MŁyniec A., Mazur L., Tomaszewski K.A., Uhl T., 2015a, Viscoelasticity and failure of collagen nanofibrils: 3D Coarse-Grained simulation studies, Soft Materials, 13, 1, 47-58

13. MŁyniec A., Tomaszewski K.A., Spiesz E.M., Uhl T., 2015b, Molecular-based nonlinear viscoelastic chemomechanical model incorporating thermal denaturation kinetics of collagen fibrous biomaterials, Polymer Degradation and Stability, 119, 87-95

14. Myers K.M., Coudrillier B., Boyce B.L., Nguyen T.D., 2010, The inflation response of the posterior bovine sclera, Acta Biomaterialia, 6, 4327-4335

15. Obuchowicz R., Ekiert M., Kohut P., Holak K., Ambrozinski L., Tomaszewski K.A., Uhl T., Mlyniec A., 2019, Interfascicular matrix-mediated transverse deformation and sliding of discontinuous tendon subcomponents control the viscoelasticity and failure of tendons, Journal of the Mechanical Behavior of Biomedical Materials, 97, 238-246

16. Okotie G., Duenwald-Kuehl S., Kobayashi H., Wu M.J., Vanderby R., 2012, Tendon strain measurements with dynamic ultrasound images: evaluation of digital image correlation, Journal of Biomechanical Engineering, 134, 024504

17. Palanca M., Tozzi G., Cristofolini L., 2016 , The use of digital image correlation in the biomechanical area: a review, International Biomechanics, 3, 1, 1-21

18. Pękala P.A., Henry B.M., Ochąa A., Kopacz P., Tatoń G., MŁyniec A., Walocha J.A., Tomaszewski K.A., 2017, The twisted structure of the Achilles tendon unraveled: A detailed quantitative and qualitative anatomical investigation, Scandinavian Journal of Medicine and Science in Sports, 27, 12, 1705-1715

19. Rashid B., Destrade M., Gilchrist M.D., 2014, Mechanical characterization of brain tissue in tension at dynamic strain rates, Journal of the Mechanical Behavior of Biomedical Materials, Special Issue on Forensic Biomechanics, 33, 43-54

20. Rizzuto E.S., Carosio S., Del Prete C., 2016, Characterization of a digital image correlation system for dynamic strain measurements of small biological tissues, Experimental Techniques, 40, $2,743-753$

21. Takaza M., Moerman K.M., Simms C.K., 2013, Passive skeletal muscle response to impact loading: experimental testing and inverse modeling, Journal of the Mechanical Behavior of Biomedical Materials, 27, 214-225

22. Tan T., Davis F.M., Gruber D.D., Massengill J.C., Robertson J.L., De Vita R., 2015, Histo-mechanical properties of the swine cardinal and uterosacral ligaments, Journal of the Mechanical Behavior of Biomedical Materials, 42, 129-137

23. Untaroiu C.D., Lu Y.C., Siripurapu S.K., Kemper A.R., 2015, Modeling the biomechanical and injury response of human liver parenchyma under tensile loading, Journal of the Mechanical Behavior of Biomedical Materials, 41, 280-291

24. Wren T., Yerby S.A., Beaupré G.S., Carter D.R., 2001, Mechanical properties of the human achilles tendon, Clinical Biomechanics, 16, 3, 245-251

25. ZAPPA E., HASheminejad N., 2017, Digital image correlation technique in dynamic applications on deformable targets, Experimental Techniques, 41, 4, 377-387 